\title{
Determination of gentamicin in different matrices by a new sensitive high-performance liquid chromatography-mass spectrometric method
}

\author{
Concepción Lecároz ${ }^{1}$, Miguel A. Campanero ${ }^{2}$, Carlos Gamazo $^{1}$ and María J. Blanco-Prieto ${ }^{3 *}$ \\ ${ }^{1}$ Department of Microbiology, University of Navarra, Pamplona, Spain; ${ }^{2}$ Department of Clinical Pharmacology, \\ Clínica Universitaria, Pamplona, Spain; ${ }^{3}$ Department of Pharmacy and Pharmaceutical Technology, \\ University of Navarra, 31080 Pamplona, Spain
}

Received 3 April 2006; returned 8 May 2006; revised 23 May 2006; accepted 30 May 2006

\begin{abstract}
Objectives: The aim of this work was to develop and validate an HPLC method for gentamicin quantification in different types of biological samples such as animal tissues and cellular material and also in pharmaceuticals.

Methods: Poly(lactide-co-glycolide) microparticles (MP) of gentamicin (PLGA 502H MP), THP-1 cells, and plasma and tissue samples of mice treated with the antibiotic either free or loaded into PLGA 502H MP were processed by a simple preparation procedure, subjected to chromatography on a reversed-phase column and measured by mass spectrometry detection. The developed method was compared with bioassay and fluorimetric assay methods previously used for gentamicin determination.

Results: The HPLC method was linear over the ranges $40-800 \mathrm{ng} / \mathrm{mL}$ and $0.1-100 \mu \mathrm{g} / \mathrm{mL}$ and showed good accuracy (average accuracy $<5.59 \%$ ) and reproducibility (CV $<6.13 \%)$. Encapsulation of gentamicin in PLGA 502H MP was determined by the three methods. Good correlation was observed between bioassay (reference method) and HPLC. Extra- and intracellular in vitro antibiotic accumulation was determined by bioassay and chromatography. Both methods gave similar extracellular concentrations but the HPLC-MS technique demonstrated an improved accuracy (5.59\% versus $14 \%)$ and precision $(6.13 \%$ versus $15 \%)$ compared with bioassay. However, only the HPLC-MS method was sensitive enough to detect the drug, intracellularly and in tissues.

Conclusions: All these data favour the use of chromatography-mass spectrometry as a versatile technique not only suitable for gentamicin quantification loaded in drug delivery systems, but also sensitive and specific enough for in vivo and intracellular studies.
\end{abstract}

Keywords: HPLC-MS, bioassay, fluorimetric assay, cellular quantification, biological matrices, drug delivery systems, PLGA

\section{Introduction}

Gentamicin is one of the most commonly used antibiotics worldwide in the treatment of many susceptible Gram-negative bacterial infections. However, it induces nephrotoxicity and ototoxicity and has a poor cellular penetration that limits its activity against intracellular pathogens, ${ }^{1}$ such as Brucella. To overcome this problem, polymeric drug delivery systems containing gentamicin have been developed with the aim of permitting the antibiotic to reach therapeutic concentrations intracellularly. Therefore, gentamicin needs to be quantified for drug delivery system characterization, intracellular distribution studies, free or vehicular, and for pharmacokinetic assays. For all these studies the most suitable quantification method must be chosen. It should have a high sensitivity, specificity and reproducibility, and should be capable of measuring a wide range of concentrations of this antibiotic, besides its low cost.

Many techniques have been developed for this purpose. Derivatization and further fluorimetric ${ }^{2,3}$ or colorimetric assays ${ }^{4}$ have been used for its quantification in solutions. Although they are easy to perform and inexpensive, the sensitivity of these techniques is too low $(0.39 \mu \mathrm{g} / \mathrm{mL}$ for gentamicin derivatization with ninhydrin $^{4}$ ) or $1 \mu \mathrm{g} / \mathrm{mL}$ when reacted with $o$-phthalaldehyde ${ }^{5}$ for intracellular concentration and pharmacokinetic studies. The

*Corresponding author. Tel: +34-948-425600; Fax: +34-948-425649; E-mail: mjblanco@unav.es 


\section{Lecároz et al.}

bioassay is the method prescribed by British, European and US pharmacopoeias for the determination of gentamicin and the other aminoglycosides in pharmaceuticals. Nevertheless, this technique has many important drawbacks. It is inaccurate, ${ }^{6}$ lacks specificity because other antibacterial substances present in biological samples can interfere in bacterial inhibition and also shows low sensitivity with detection limits around $0.1 \mu \mathrm{g} /$ $\mathrm{mL}$, which hinder gentamicin quantification in pharmacokinetic or cellular internalization studies. ${ }^{7}$

Automated assay procedures, such as radioimmunoassay (RIA), enzyme immunoassays (EIA) (heterogeneous or homogeneous), fluoroimmunoassay, direct chemiluminescence immunoassay and nephelometric and turbidimetric assay, ${ }^{6}$ have been developed for gentamicin quantification in serological samples. They are very sensitive, specific, require only small sample volume and can be performed by commercially available kits. However, these automated assays show a series of drawbacks: the requirement for expensive $\beta$-counting equipment, provision for use and disposal of radioactive materials, a labour-intensive procedure $^{8}$ and skilled personnel.

In previous work performed to date in our laboratory, the chosen technique was derivatization with $o$-phthalaldehyde and fluorimetric assay, ${ }^{9}$ which was performed for gentamicin encapsulation in a drug delivery system. Its drawbacks, as described above, are the low specificity and the relatively low sensitivity. The aim of the present work was to develop a technique suitable for gentamicin quantification in cellular internalization studies, which can be also applied for pharmacokinetic studies and for the characterization of particulated systems (gentamicin-loading, antibiotic in vitro release). A more sensitive method was needed, as long as low concentrations could be found in biological samples, and, at the same time, minimal interferences were desirable with proteins and other components of samples. HPLC is the technique that offers the highest resolution and best degree of specificity. When coupled with mass spectrometry, it becomes the most powerful method for the confirmation of antibiotic residues in food, ${ }^{6}$ with quantification limits in the order of nanograms. The technique developed in the present work is a sensitive analytical method based on a highly specific and selective chromatographic separation and mass spectrometry detection after electrospray ionization (HPLC-ESI-MS). Furthermore, comparisons with fluorimetric assay after drug derivatization (the technique used up to now) and bioassay (as reference method) were performed.

\section{Materials and methods}

\section{Materials}

Gentamicin sulphate, $\beta$-mercaptoethanol, Trypan Blue and phorbol 12-myristate 13-acetate (PMA) were from Sigma-Aldrich Co. (St Louis, MO, USA).

The poly(D, L-lactide-co-glycolide) (PLGA) copolymer with free carboxyl end group: 13.7 kDa, PLGA 50:50 (Resomer ${ }^{\circledR}$ RG 502H) was provided by Boehringer-Ingelheim (Ingelheim, Germany). Ethyl acetate, monopotassium phosphate, sodium dibasic phosphate, diethyl ether, boric acid, ethanol, $\mathrm{NaOH}$, trichloroacetic acid and dichloromethane were from Panreac (Barcelona, Spain). Poly(vinylalcohol) (PVA, MW 15000) was purchased from BDH Supplies (Poole, UK). Pentafluoropropionic acid (PFPA) was from Fluka Chemie (Buchs, Switzerland). Tobramycin (internal standard), procured as Tobra-Gobens ${ }^{\circledR}$ (the brand name of the product registered for clinical use in Spain), was purchased from Normon (Madrid, Spain). Methanol (Lichrosolv ${ }^{\circledR}$ ) and $o$-phthalaldehyde were from Merck (Darmstadt, Germany). Mueller-Hinton broth was purchased from DIFCO Laboratories (Detroit, MI, USA) and the American Bacteriologic Agar from Pronadisa, Hispanlab (Madrid, Spain). Sterile swabs (Invasive sterile Eurotubo collection swabs) were from I.A.S.A. (Rubi, Spain), and sterile cellulose discs were purchased from BD (Franklin Lakes, NJ, USA). Staphylococcus aureus 25923 was obtained from the American Type Culture Collection $\left(\mathrm{ATCC}^{\circledR}\right)$.

Finally, for cell culture, THP-1 cells, a human myelomonocytic cell line displaying macrophage-like activity, ${ }^{10}$ were kindly provided by Professor D. Raoult of the Unite des Rickettsies, Université de la Mediterranée, Marseille, France. RPMI cell culture medium and L-glutamine were purchased from Gibco (Invitrogen, Carlsbad, CA, USA). Fetal calf serum was from Biochrom AG (Berlin, Germany).

\section{Preparation and physicochemical characterization of gentamicin microparticles}

Poly(lactide-co-glycolide) microparticles (MP), made of PLGA 502H, and containing gentamicin, were prepared by a water-oil-water solvent evaporation technique. ${ }^{11}$ Briefly, the antibiotic $(10 \mathrm{mg})$ dissolved in $0.5 \%$ PVA in phosphate buffer, $\mathrm{pH} 6.0$, and $200 \mathrm{mg}$ of polymer dissolved in dichloromethane were mixed by ultrasonication (Branson sonifier 450, Branson Ultrasonics corp., Danbury, CT, USA) under cooling for $1 \mathrm{~min}$, to form a $\mathrm{W}_{1} / \mathrm{O}$ emulsion. This inner emulsion was added to $2 \mathrm{~mL}$ of $1 \%$ PVA $\left(\mathrm{W}_{2}\right)$ and homogenized again by ultrasonication. The resulting $\left(\mathrm{W}_{1} / \mathrm{O}\right) \mathrm{W}_{2}$ emulsion was poured into $50 \mathrm{~mL}$ of $0.2 \%$ PVA and continuously stirred for, at least, $3 \mathrm{~h}$ at room temperature to allow solvent evaporation and MP formation. After preparation, the microspheres were isolated by centrifugation (7000 g, $5 \mathrm{~min})$, washed with ultra pure water and freeze-dried.

MP diameter was determined by laser light diffraction (Mastersizer ${ }^{\circledR}$, Malvern Instruments, Worcestershire, UK). The average particle size was expressed as the volume mean diameter in micrometres. The zeta potential of the MP dispersed in ultra pure water was performed using a Zetamaster system (Malvern Instruments, Worcestershire, UK).

The final formulation containing gentamicin presented a mean particle diameter of $1.8 \mu \mathrm{m}$ and zeta potential of $-21.5 \mathrm{mV}$.

\section{Antibiotic extraction from particles}

Antibiotic extraction from MP was performed as follows:

(i) Dissolving the loaded microspheres in $1 \mathrm{~mL}$ of dichloromethane and extracting the drug three times with $2 \mathrm{~mL}$ of phosphate buffer, $\mathrm{pH} 6.0$.

(ii) Dispersing the microspheres in $1 \mathrm{~mL}$ of $0.1 \mathrm{M} \mathrm{NaOH}$.

In both cases, samples were centrifuged and gentamicin was quantified in the supernatant.

\section{Determination of cellular antibiotic accumulation}

THP-1 cells (human myelomonocytic cell line displaying macrophage-like activity $)^{10}$ were maintained in RPMI medium supplemented with $10 \%$ heat inactivated fetal calf serum and $2 \mathrm{mM}$ L-glutamine in a $5 \% \mathrm{CO}_{2}$ atmosphere. During culture, cell density did not exceed $1 \times 10^{6}$ cells $/ \mathrm{mL}$. A volume of $500 \mu \mathrm{L}$ of THP- 1 monocytes was plated into wells $\left(1 \times 10^{6}\right.$ cells $\left./ \mathrm{mL}\right)$ with $10 \mathrm{ng} / \mathrm{mL}$ PMA and 


\section{HPLC-MS gentamicin determination in different matrices}

incubated for $48 \mathrm{~h}^{12}$ in order to allow differentiation into macrophages. Then, wells were washed once, and different concentrations of gentamicin were added in fresh medium and incubated for $24 \mathrm{~h}$. Afterwards, extracellular fractions were collected; cells were washed five times to eliminate the antibiotic outside the cells and detached from wells with $500 \mu \mathrm{L}$ of $0.05 \%$ trypsin-EDTA. Finally, three cycles of freeze-thawing (in liquid nitrogen and water bath at $45^{\circ} \mathrm{C}$ ) were performed to assure complete cell lysis, and intracellular content was collected after centrifugation $(1000 \mathrm{~g}, 10 \mathrm{~min})$ to remove cellular debris. All samples were then frozen $\left(-20^{\circ} \mathrm{C}\right)$ until analysis. For the present study, only HPLC and bioassay were used for gentamicin quantification. The fluorimetric assay could not be used because of the interference in the analysis by amino acids present in RPMI.

\section{Quantification of gentamicin concentrations in tissue samples}

Tissue concentrations of gentamicin administered either free or loaded into PLGA 502H MP were determined in mice. Female $\mathrm{BALB} / \mathrm{c}$ mice of $20 \pm 1 \mathrm{~g}$ were supplied by Harlan Iterfauna Ibérica (Barcelona, Spain). The experiments were performed in compliance with the regulations of the responsible committee of the University of Navarra in line with the European legislation on animal experiments (86/609/EU).

Mice $(n=3)$ received one dose of $30 \mu \mathrm{g}(1.5 \mathrm{mg} / \mathrm{kg})$ of the antibiotic intravenously, either free or loaded into PLGA particles. After the administration of the free antibiotic, concentrations were undetectable. Consequently, three doses every $2.5 \mathrm{~h}$ of $40 \mathrm{mg} / \mathrm{kg}$ of free gentamicin were used. At different times, animals were anaesthetized, bled by retro-orbital puncture and sacrificed by cervical dislocation and livers were extracted. Once diluted in saline solution, organs were homogenized, centrifuged (10000 g, $15 \mathrm{~min})$ and supernatants collected. Proteins were precipitated by $1 \%$ trichloroacetic acid and samples were analysed by HPLC as described below.

\section{Gentamicin quantification methods}

HPLC-mass spectrometry. The apparatus used for the HPLC analysis was a Model 1100 Series LC coupled with an atmospheric pressure (AP)-electrospray ionization (ESI) mass spectrometer (HP 1100 with MSD VL, Waldbronn, Germany). A Hewlett-Packard computer using the ChemStation G2171 AA program was used for data acquisition and analysis.

Separation was carried out at $50^{\circ} \mathrm{C}$ on a reversed-phase Lichrospher $\mathrm{C}_{8} 60 \mathrm{RP}$ column, of $250 \mathrm{~mm} \times 4 \mathrm{~mm}$, purchased from Merck (Darmstadt, Germany). The chosen flow rate was $0.5 \mathrm{~mL} / \mathrm{min}$. Ionization was performed in positive Selected Ion Monitoring (SIM) mode; the ions $\mathrm{m} / \mathrm{z}$ values were 468.20 for gentamicin and 478.30 for tobramycin. Capillary voltage was $4000 \mathrm{~V}$ and fragmentator voltage was $140 \mathrm{~V}$. Temperature was $350^{\circ} \mathrm{C}$, and the drying gas $(12 \mathrm{~L} / \mathrm{min})$ and the nebulizer gas $(60$ psi) were both nitrogen. As mobile phase, a linear gradient of PFPA (20 mM in ultrapure water) and methanol was used. For the chromatographic quantification of gentamicin, all samples, from MP extraction or from cell studies, were processed equally (diluted with the same volume of a solution with a proportion of PFPA:methanol 1:1) and either 1 or $25 \mu \mathrm{L}$ was injected onto the HPLC column, maintaining the injected tobramycin amount constant.

Bioassay. Gentamicin was assayed by a standard disc-diffusion technique. Two or three isolated colonies of $S$. aureus were inoculated in fresh Mueller-Hinton broth and incubated at $37^{\circ} \mathrm{C}$ until reaching an absorbance at $590 \mathrm{~nm}$ of $0.125\left(10^{8} \mathrm{cfu} / \mathrm{mL}\right)$. A dilution of this culture $\left(10^{5} \mathrm{cfu} / \mathrm{mL}\right)$ was used to inoculate Mueller-Hinton agar plates for confluent growth of bacteria. Running in parallel, $20 \mu \mathrm{L}$ of different standard concentrations of gentamicin and samples were added to cellulose discs and incubated for $5 \mathrm{~h}$ at $37^{\circ} \mathrm{C}$. The discs were placed on the inoculated Mueller-Hinton agar plates and incubated for $24 \mathrm{~h}$ at $37^{\circ} \mathrm{C}$. Finally, inhibition diameters were measured and the unknown concentrations were determined extrapolating the diameter values in the standard exponential curve.

Derivatization and fluorimetric assay. Standard concentrations of gentamicin were prepared by serial dilutions in boric acid $0.4 \mathrm{M}$, $\mathrm{pH}$ 9.7, and added to a 96-well microplate (TPP, Trasadingen, Switzerland). Samples were also diluted, when needed, in boric acid. Then, fluorimetric assay solution $[0.04 \% o$-phthalaldehyde, $0.1 \%(\mathrm{v} / \mathrm{v})$ diethyl ether, $0.2 \%(\mathrm{v} / \mathrm{v}) \beta$-mercaptoethanol in boric acid] was added to the standard solutions. Fluorescence was measured, immediately, in a Tecan GENios fluorimeter (Tecan Group Ltd, Maennedorf, Switzerland) (excitation $340 \mathrm{~nm}$, emission $450 \mathrm{~nm}$ ) and sample concentrations were calculated by means of the standard curve. 5

\section{Statistics}

For determination of known concentrations of gentamicin by the three techniques, linear regression was performed by using standard techniques at four antibiotic concentrations, with three different samples measured at each concentration level.

In the case of antibiotic quantification from MP, since the three methods were compared, analysis of variance (ANOVA) was performed and Tukey was used as the post-hoc test. For intracellular accumulation study, Student's $t$-test was performed because HPLC and bioassay were compared. In all cases, bioassay was used as the reference method. For all statistical analysis, SPSS program, version 11.0 (SPSS Inc., Chicago, USA), was used.

\section{Results}

\section{Determination of gentamicin by HPLC}

Gentamicin and tobramycin were found to have retention times of 9.42 and $8.66 \mathrm{~min}$, respectively. Typical chromatograms of a pharmaceutical formulation, cell extracts and mice liver are shown in Figure $1(\mathrm{a}-\mathrm{c})$. Good separation was obtained from the endogenous background, and area peak values were used for antibiotic quantification. Standard curves were obtained at two different ranges of concentration: for gentamicin extraction from MP and for extracellular gentamicin concentrations, $1 \mu \mathrm{L}$ was injected and $100,50,25,12.5,6.25,3.125,1$ and $0.1 \mu \mathrm{g} / \mathrm{mL}$ standards were used, and for tissue and intracellular antibiotic quantification, $25 \mu \mathrm{L}$ was injected and samples with $8,4,2,1$, $0.5,0.25,0.125$ and $0.04 \mu \mathrm{g} / \mathrm{mL}$ gentamicin concentrations were used as standards. A linear relationship was found between the peak area of gentamicin divided by the area of tobramycin (the internal standard) and gentamicin concentration, giving standard curves with $r^{2}>0.99$. The method showed good levels of precision over the 2 years that it has been used, as demonstrated by the relatively small $\mathrm{CVs}$ at each concentration (average $\mathrm{CV}$ for interday variability $<6.13 \%$ ). Moreover, an excellent agreement between the measured concentrations and the spiked concentrations was obtained (average accuracy between 0.04 and $100 \mu \mathrm{g}$ / $\mathrm{mL}$, concentration range $<5.59 \%$ ).

\section{Determination of gentamicin by bioassay}

The inhibition zones were clearly defined. The concentrations used in the standard curve were 2000, 1000, 800, 660, 500, 250, 


\section{Lecároz et al.}
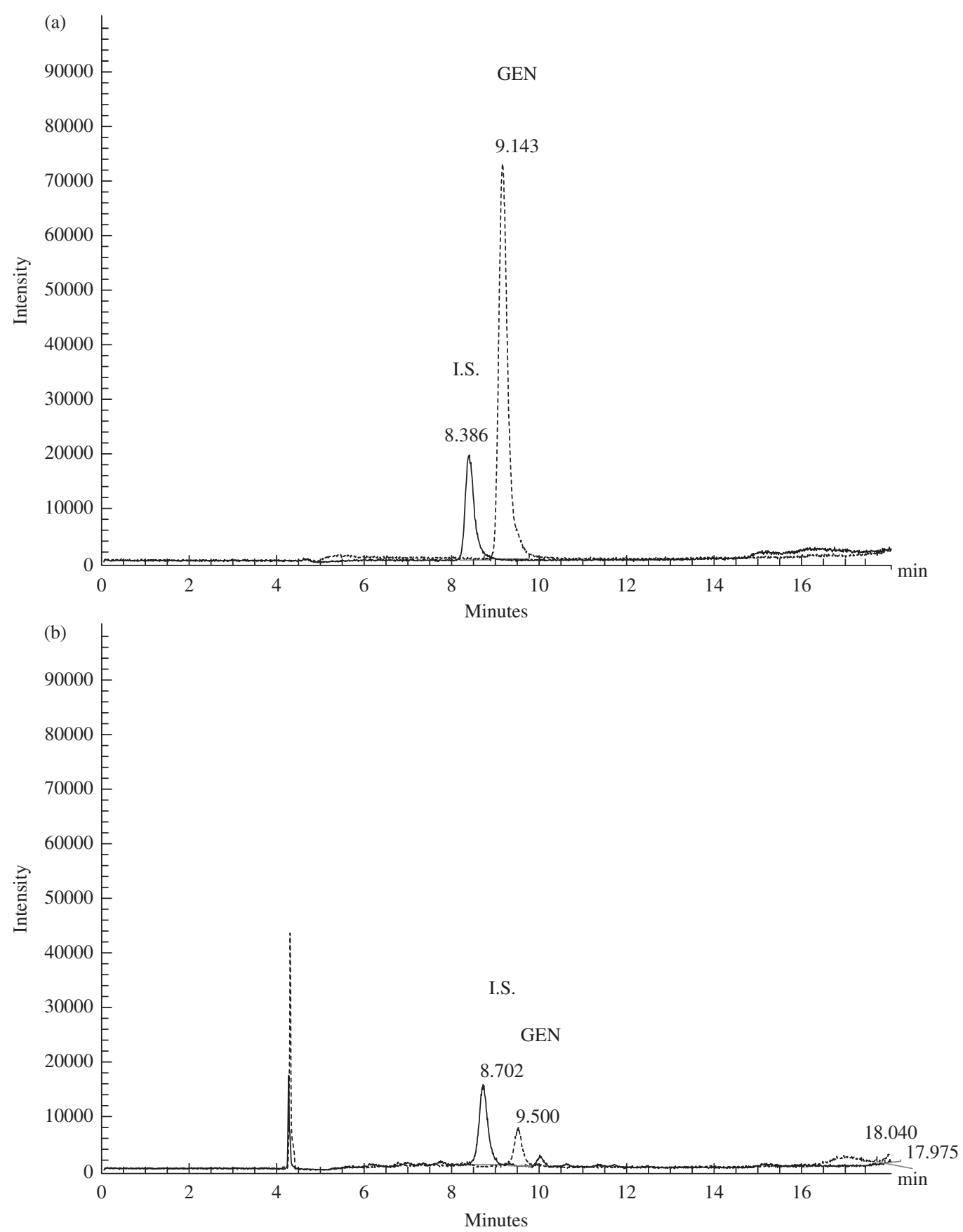

Figure 1. Typical chromatograms of gentamicin (GEN) and tobramycin as internal standard (I.S.). (a) PLGA $502 \mathrm{HMP}$ (gentamicin concentration: $86 \mu \mathrm{g} / \mathrm{mL}, 1 \mu \mathrm{L}$ of sample injected). (b) Intracellular gentamicin concentration after THP-1 cell incubation with $1250 \mu \mathrm{g} / \mathrm{mL}$ of the antibiotic (gentamicin concentration: $0.48 \mu \mathrm{g} / \mathrm{mL}$, $25 \mu \mathrm{L}$ of sample injected). (c) Liver concentration of gentamicin at 7 days post-administration of MP of PLGA $502 \mathrm{H}$ (gentamicin concentration: $0.16 \mu \mathrm{g} / \mathrm{mL}, 25 \mu \mathrm{L}$ of sample injected). A colour version of this figure is available as Supplementary data at JAC Online (http://jac.oxfordjournals.org/).

$125,74,62,31$ and $25 \mu \mathrm{g} / \mathrm{mL}$ and an exponential relationship was found between the inhibition diameter and antibiotic concentration with $r^{2}>0.98$. Intraday variability, defined as $\mathrm{CV}$ assayed by $2000,500,125$ and $62 \mu \mathrm{g} / \mathrm{mL}$ samples, averaged $<3 \%$. The interday variability was determined by the average $\mathrm{CV}$ of assays of five different days and the result was $<15 \%$. The assay was sensitive above $25 \mu \mathrm{g} / \mathrm{mL}$ of the antibiotic (LOQ value). To determine accuracy, two spiked controls of 500 and $100 \mu \mathrm{g} / \mathrm{mL}$ were assayed three times. The values obtained were averaged and correlated to the expected value. An accuracy of $14 \%$ was obtained.

\section{Determination of gentamicin by fluorescence assay}

Concentrations of gentamicin chosen for the standard curve were $1000,500,250,125,62.5,31.5,15.62,8.71$ and $3.9 \mu \mathrm{g} / \mathrm{mL}$. Concentrations of the antibiotic and fluorescence emission read at $450 \mathrm{~nm}$ presented a linear relationship with $r^{2}<0.98$. 


\section{HPLC-MS gentamicin determination in different matrices}

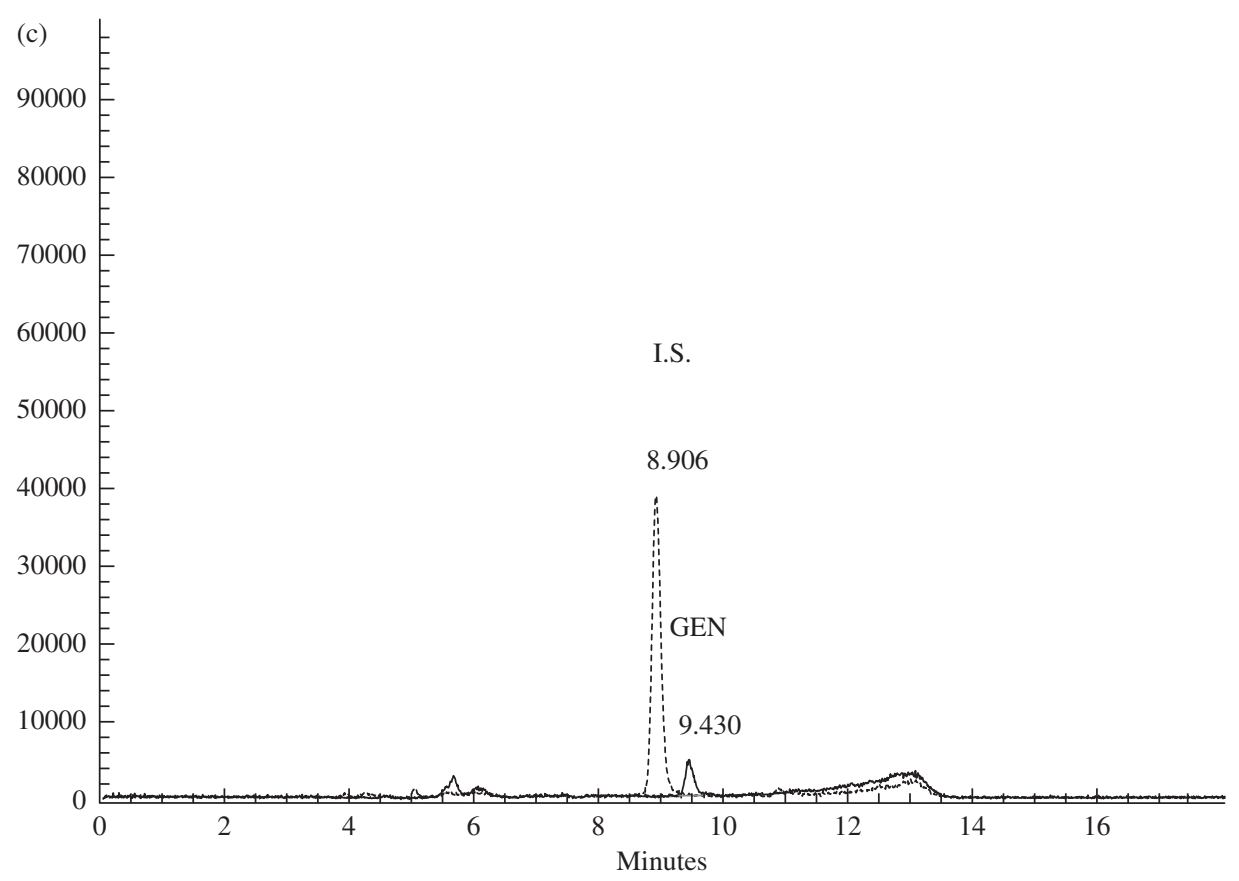

Figure 1. Continued

Intraday variability was $<12 \%$, while interday variability reached $39 \%$. The sensitivity of the assay was $3.9 \mu \mathrm{g} / \mathrm{mL}$.

\section{Comparison of HPLC, bioassay and fluorescence assays}

Determination of gentamicin from microparticles. As shown in Figure 2(a), gentamicin encapsulation obtained with fluorescence assay always had lower results than the other two techniques. Statistical differences were observed when comparing antibiotic derivatization with chromatography. This statistical significance was higher when $\mathrm{NaOH}$ was used for extraction. On the contrary, HPLC and bioassay presented no differences in drug quantification.

Linear regressions were also performed to compare both extraction techniques by the three methods. In concordance with the results of Figure 2, the good correlation between HPLC and bioassay was proved once more in all cases: for dichloromethane and $\mathrm{NaOH}$ extraction, $r^{2}$ values of 0.889 and 0.855 , respectively, were obtained. On the contrary, poor correlation was observed between fluorimetric assay and bioassay, with $r^{2}$ of 0.543 for dichloromethane extraction and even lower $\left(r^{2}=\right.$ 0.266 ) for $\mathrm{NaOH}$.

Determination of cellular gentamicin accumulation. Gentamicin intracellular and extracellular concentrations were determined by HPLC and bioassay (Figure 2b). Antibiotic derivatization with fluorimetric assay could not be used because of interference with cell culture medium. Comparing the three methods, only chromatography was sensitive enough for detecting intracellular concentrations of the polar antibiotic, because only a small amount of it crosses the cell membrane, while bioassay could only quantify the extracellular gentamicin. For these latter concentrations of the aminoglycoside, both techniques gave similar results, but the deviations obtained with HPLC were smaller than those with the microbiological assay (Figure 2b).
Finally, the scatterplot (Figure 3) showed the agreement between HPLC and bioassay with an $r^{2}$ of 0.954, indicating the good correlation between the two methods.

Quantification of gentamicin concentrations in tissue samples. HPLC proved to be the only technique sensitive enough to detect gentamicin concentrations in animal organs after both gentamicin-free and gentamicin-encapsulated administration to mice. The detection limit was similar to that observed for particle extraction $(0.02 \mu \mathrm{g} / \mathrm{mL}$ or $0.13 \mu \mathrm{g} / \mathrm{g}$ tissue) and lower than bioassay $(25 \mu \mathrm{g} /$ $\mathrm{mL}$ ). Comparison between HPLC and bioassay was not performed since the levels of gentamicin detected in tissues were below the quantification limit of bioassay. As shown in Figure 4, the developed method allowed gentamicin quantification of both the free and released antibiotic from MP. Free gentamicin reached a concentration peak of $1.6 \mu \mathrm{g} / \mathrm{g}$ within the first day post-administration and was cleared from hepatic tissue in 4 days. On the contrary, microencapsulation led to a controlled release of the drug as reflected in sustained concentrations detected in liver (Figure 4), with measurable gentamicin levels for 27 days.

\section{Discussion}

The objective of the present study was to develop an HPLC assay for gentamicin quantification that allows low concentration analysis in cell cultures and tissues as an alternative to the methods employed today. With this purpose, the present work compares an HPLC method with bioassay and derivatization with a fluorinated compound for the determination of gentamicin levels in cell internalization studies, pharmacokinetic studies and new microsize pharmaceutical preparations by direct injection.

Aminoglycosides are traditionally difficult to determine with conventional HPLC because of the lack of strong chromophores in the molecule structure and their highly polar characteristics. 


\section{Lecároz et al.}

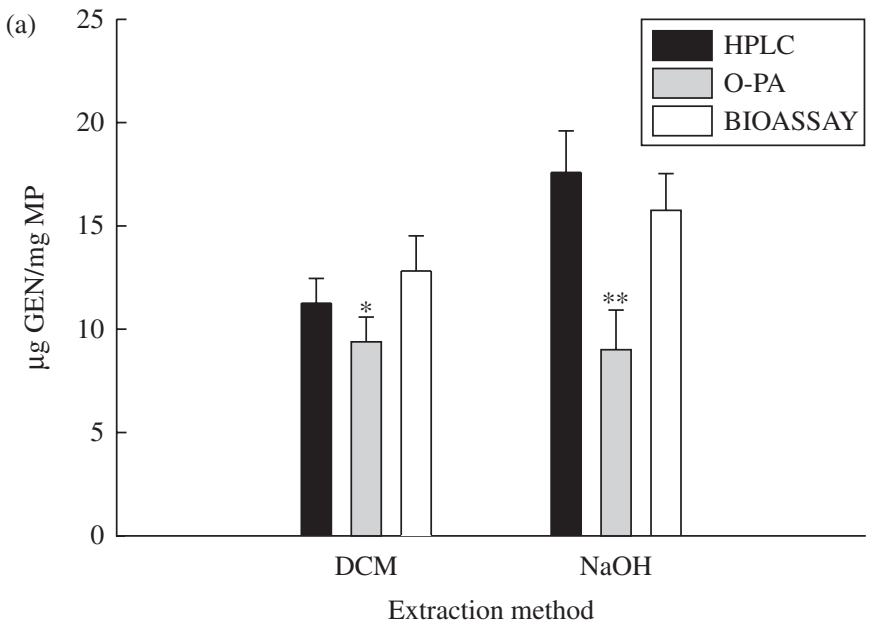

(b)

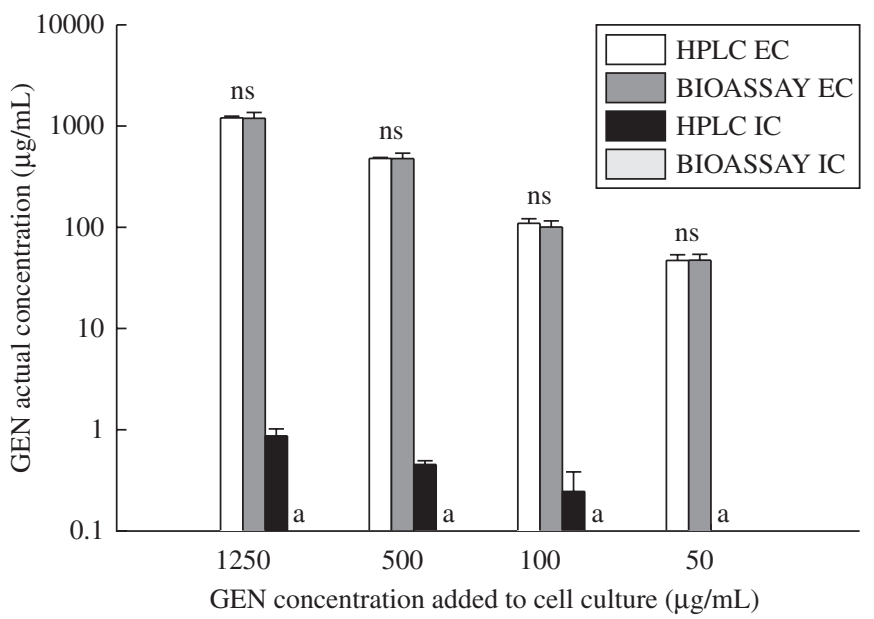

Figure 2. Comparison between methods. (a) Comparison of the three methods for quantification of gentamicin (GEN) extraction from PLGA 50:50 MP by $\mathrm{NaOH}$ and dichloromethane (DCM). (b) Comparison of HPLC and bioassay for gentamicin intracellular (IC) and extracellular (EC) determination, after incubating the antibiotic with THP-1 cells for $24 \mathrm{~h}$. Bars represent the means \pm SD of four samples. For comparison of three methods (a) analysis of variance (ANOVA) was performed and Tukey was performed as the post-hoc test, and to compare two methods (b) Student's $t$-test was performed: $* P<0.05$; $* * P<$ 0.01; ns, not significant; a, not detected.

In general terms, derivatization with a fluorescent reagent, such as fluorimetric assay, has been adopted as the methodology of choice for the analysis of gentamicin. ${ }^{13}$ However, the use of this methodology is not free from drawbacks. The fluorimetric assay reagent is unstable and has to be prepared regularly, and an incomplete derivatization of gentamicin was observed when certain samples (i.e. organic samples) were processed. ${ }^{14}$ This could be an important source of error in the method, leading to the underestimation of the aminoglycoside in the samples. This problem can be resolved by using more specific liquid chromatographic/mass spectrometric (LC/MS) techniques.

Gentamicin and other aminoglycosides are extremely hydrophilic, due to the high number of amino and hydroxyl moieties on the carbon chain. Therefore, they are positively charged at the $\mathrm{pH}$ range employed in reversed phase-liquid chromatography and are

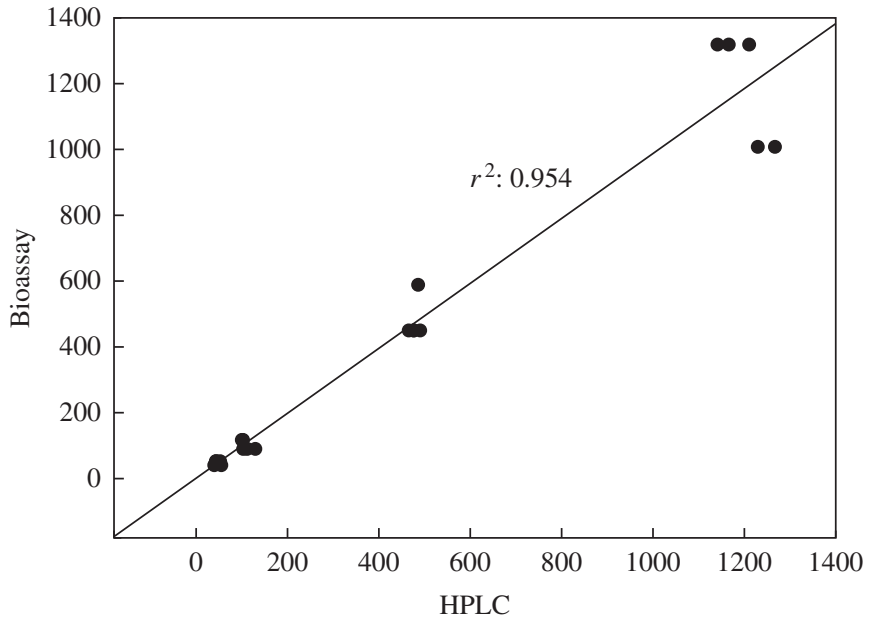

Figure 3. Scatterplot of extracellular gentamicin concentrations determined by bioassay and HPLC, after cell incubation with $1250,500,100$ and $50 \mu \mathrm{g} / \mathrm{mL}$.

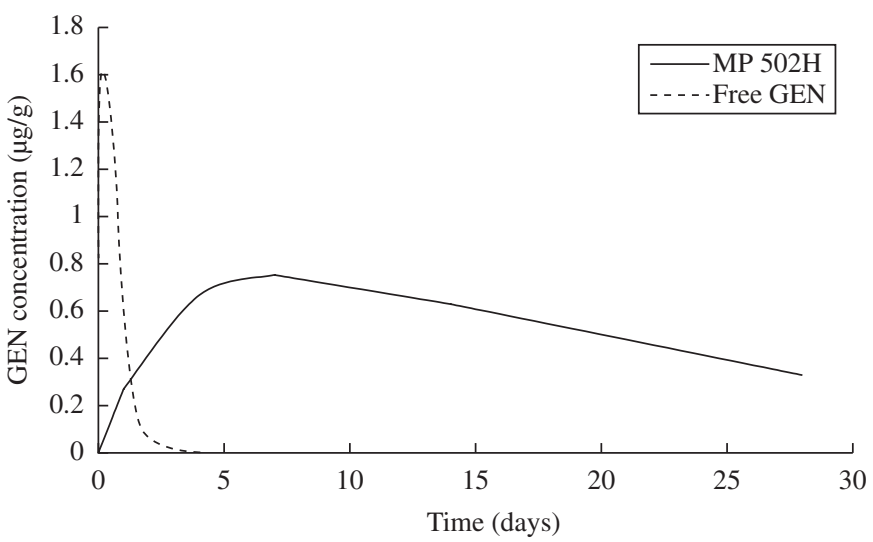

Figure 4. Determination of gentamicin (GEN) concentrations in mouse hepatic tissue, performed by HPLC. Free gentamicin (Free GEN) (three doses every $2.5 \mathrm{~h}$ of $40 \mathrm{mg} / \mathrm{kg}$ ) and microencapsulated antibiotic into 502H MP (MP 502H) (one dose of $1.5 \mathrm{mg} / \mathrm{kg}$ ) were administered intravenously and at different times, animals were sacrificed and gentamicin was determined in extracted liver.

not retained on conventional C-18 bonded silica columns without an ion pair agent, but these agents can suppress ionization and foul the LC/MS interface. The use of a fluorinated ion pair reagent with moderate volatility, PFPA, and the application of a step gradient with a decreasing concentration of the reagent (gentamicin is therefore eluted into the mass spectrometer at nearly zero PFPA concentration), allows this problem to be resolved. In fact, the obtained limit of quantification $(0.04 \mu \mathrm{g} /$ $\mathrm{mL}$ ) was still more than adequate for monitoring the gentamicin concentration in samples and was less than that of both the bioassay $(25 \mu \mathrm{g} / \mathrm{mL})$ and fluorimetric assay $(3.9 \mu \mathrm{g} / \mathrm{mL})$.

Agar well-based microbiological methods are traditionally employed to determine antimicrobial concentrations by measuring graded responses to known concentrations of an antimicrobial against a standard curve. Bioassays are easy to perform and do not require special equipment. However, chromatographic assays are preferred to biological assays for antibiotic quantification. In our case, the major advantages of the HPLC method compared 


\section{HPLC-MS gentamicin determination in different matrices}

with the bioassay are its enhanced accuracy (5.59\% versus $14 \%)$ and precision $(6.13 \%$ versus $15 \%)$.

We have compared the results of the HPLC analysis of the samples with those obtained after microbiological assay and the application of the derivatization procedure. Cell studies showed an excellent correlation between the gentamicin concentrations measured by HPLC and those measured by bioassay. However, the poor sensitivity of the bioassay compared with the HPLC method impaired its widespread use for the determination of the intracellular antibiotic concentrations. Studies in our laboratories have shown that a better fit of the extracellular data was obtained when both techniques were applied to determine gentamicin. Similar results were obtained comparing the results of the HPLC assay and the bioassay of extracted samples from particles. However, the majority of data points fell significantly below the line of unity and showed a poor overall fit to the data when fluorimetric analysis and HPLC were compared. These results suggest that the derivatization procedure underestimates the gentamicin concentration of the analysed sample.

In addition, for gentamicin determination in animal tissues, the chromatographic technique was the only assay able to quantify gentamicin below $1 \mu \mathrm{g} / \mathrm{mL}$, showing, once more, a clear advantage over the other two techniques. Furthermore, since the biological samples are known to be a complex matrix containing proteins and other compounds, a specific method becomes necessary.

In summary, we have developed an HPLC-MS method adequate for determining gentamicin in biological samples and pharmaceutical preparations. In contrast to other procedures, the developed technique is rapid and precise and ensures high specificity, being a very attractive alternative to the microbiological assays employed today.

\section{Acknowledgements}

This work was supported by the Program 'Redes Temáticas de Investigación Cooperativa del FIS-Brucellosis (ref. no. G03/ 201)' and 'Proyectos de Investigación Universidad de Navarra' (PIUNA). Fellowship support for C. L. from 'Gobierno de Navarra' and 'Asociación de Amigos' are gratefully acknowledged.

\section{Transparency declarations}

None to declare.

\section{Supplementary data}

A colour version of Figure 1 is available as Supplementary data at JAC Online (http://jac.oxfordjournals.org/).

\section{References}

1. Gonzalez LS, Spencer JP. Aminoglycosides: a practical review. Am Fam Physician 1998; 58: 1811-20.

2. Hascicek C, Gonul N, Erk N. Mucoadhesive microspheres containing gentamicin sulfate for nasal administration: preparation and in vitro characterization. Farmaco 2003; 58: 11-6.

3. Xue JM, Shi M. PLGA/mesoporous silica hybrid structure for controlled drug release. J Control Release 2004; 98: 209-17.

4. Frutos $\mathrm{P}$, Torrado SM, Perez-Lorenzo $\mathrm{E}$ et al. A validated quantitative colorimetric assay for gentamicin. $J$ Pharm Biomed Anal 2000; 21: 1149-59.

5. Benson JR, Hare PE. O-phthalaldehyde: fluorogenic detection of primary amines in the picomole range. Comparison with fluorescamine and ninhydrin. Proc Natl Acad Sci USA 1975; 72: 619-22.

6. Stead DA. Current methodologies for the analysis of aminoglycosides. J Chromatogr B Biomed Sci Appl 2000; 747: 69-93.

7. Teixeira LR, Sasse SA, Villarino MA et al. Antibiotic levels in empyemic pleural fluid. Chest 2000; 117: 1734-9.

8. Delaney CJ, Opheim KE, Smith AL et al. Performance characteristics of bioassay, radioenzymatic assay, homogeneous enzyme immunoassay, and high-performance liquid chromatographic determination of serum gentamicin. Antimicrob Agents Chemother 1982; 21: 19-25.

9. Prior S, Gander B, Blarer N et al. In vitro phagocytosis and monocyte-macrophage activation with poly(lactide) and poly(lactide-coglycolide) microspheres. Eur J Pharm Sci 2002; 15: 197-207.

10. Tsuchiya S, Yamabe M, Yamaguchi $Y$ et al. Establishment and characterization of a human acute monocytic leukemia cell line (THP-1). Int J Cancer 1980; 26: 171-6.

11. Blanco-Prieto $M$, Lecaroz $C$, Renedo $M$ et al. In vitro evaluation of gentamicin released from microparticles. Int J Pharm 2002; 242: 203-6.

12. Dreskin SC, Thomas GW, Dale SN et al. Isoforms of Jun kinase are differentially expressed and activated in human monocyte/macrophage (THP-1) cells. J Immunol 2001; 166: 5646-53.

13. British-Pharmacological-Society. British Pharmacopoeia 2004. London, UK: The Stationery Office Books, 2004.

14. Chissell JF, Freeman M, Loran JS et al. British pharmacopoeial gentamicin sulphate component ratio test by high-performance liquid chromatography. The effect of derivative breakdown on final result. J Chromatogr 1986; 369: 213-7. 